\title{
Localization, Characterization and Local Biokinetics of Tattoo Pigment Particles in Human Skin and Lymph Nodes by Means of Synchrotron-based Micro- and NanoXRF
}

Christian Seim ${ }^{1}$, Ines Schreiver ${ }^{2}$, Bernhard Hesse $^{3}$, Hiram Castillo-Michel ${ }^{3}$, Julie Villanova ${ }^{3}$, Peter Laux $^{2}$, Nadine Dreiack ${ }^{2}$, Randolf Penning ${ }^{4}$, Remi Tucoulou ${ }^{3}$, Marine Cotte ${ }^{3} \&$ Andreas Luch ${ }^{2}$

1. Physikalisch-Technische Bundesanstalt, X-ray Spectrometry, Abbestr. 2-12, 10587 Berlin, Germany

2. German Federal Institute for Risk Assessment (BfR), Department of Chemical and Product Safety, Max-Dohrn-Strasse 8-10, 10589, Berlin, Germany

3. European Synchrotron Radiation Facility (ESRF), 38043, Grenoble, Cedex 9, France

4. Institute of Forensic Medicine, Ludwig-Maximilians University, Munich, Germany

* Corresponding author, christian.seim@ptb.de

Within the last twenty years, tattoos have made their way into mainstream culture, especially for young people. With $36 \%$ of people younger than 40 years having at least one tattoo, risk assessment of tattoo colorants and permanent make-up has shifted into spotlight on a global level [1]. In the past, health risk of tattoos was mostly deemed to lie in uncertain hygiene standards and the subsequent danger of acquiring a post-tattoo infection. But recently, with the increasing prevalence of tattoos, safety concerns have extended to particle distribution and their effects inside the human body. Especially the distribution and speciation of tattoo pigments and their contaminants in the human body is still poorly understood and therefore in the focus of the study presented here.

This contribution is based on [2], in which we analyzed tattooed human skin samples and regional lymph nodes from four human donors (corpses). To the best of our knowledge the speciation of $\mathrm{TiO}_{2}$, and the identities and size ranges of pigment particles migrating from subepidermal skin layers towards lymph nodes have never been analytically investigated in humans before.

Mass spectrometry methods (MS), such as inductively coupled plasma (ICP)-MS and laserdesorption/ionization time-of-flight (LDI-ToF)-MS, were employed to assess the general elemental composition of the tattoo pigments in the skin and lymph node tissue. To associate elements identified by MS (see table 1 in [2]) to a location within the tissue sections, we conducted micro X-ray fluorescence analysis ( $\mu$-XRF) and micro X-ray absorption near edge structure analysis ( $\mu$-XANES) at the ESRF at ID21 [3] and nano-XRF analysis at ID16B [4] to additionally assess particle sizes and potentially sizedependent migration of tattoo pigments. $\mu$-XRF experiments were carried out at a photon energy of 5.05 $\mathrm{keV}$ and nano-XRF experiments at $17.5 \mathrm{keV}$. Furthermore, we investigated biomolecular changes in the vicinity of tissue areas, where we found tattoo pigments, by means of synchrotron-based Fourier transform infrared ( $\mu$-FTIR) spectroscopy. Both, MS and $\mu$-FTIR spectroscopy results will not be further discussed here, but are comprehensively presented in [2].

Tattoo pigments are composed of either polyaromatic compounds or inorganic metals (and their oxides). Thus, a broad range of elements can be found in tattooed human tissue - among them nickel (Ni), chromium (Cr), manganese ( $\mathrm{Mn}$ ), and cobalt (Co) - usually as components of color-giving pigments or element contamination. After carbon black (not assessed in this study) the inorganic titanium dioxide $\left(\mathrm{TiO}_{2}\right)$ - a white pigment usually applied to create certain shades when mixed with colorants - is the second 
most commonly used ingredient of tattoo inks. Its toxicity mainly depends on its chemical speciation, which is either rutile or the photocatalytically active anatase. The latter, if exposed to sunlight, can lead to formation of reactive oxygen species [2].

$\mu$ - and nano-XRF experiments revealed that all skin and lymph node tissue samples contained $\mathrm{TiO}_{2}$ with an average particle size of $180 \mathrm{~nm} \pm 23 \mathrm{~nm}$ which suggests that transport from skin to the regional lymph nodes is not prevented by larger particle sizes. Through $\mu$-XANES analysis, we additionally found that rutile is the predominant crystal phase of $\mathrm{TiO}_{2}$ in all tattooed donors, with minor contributions of anatase. The highly brominated pigment copper phthalocyanine green 36 which was mostly localized adjacent to $\mathrm{TiO}_{2}$ particles (see fig. 1) showed a more heterogeneous size distribution. Particle sizes ranged from 50 $\mathrm{nm}$ (and potentially smaller; beam size at ID16b was $50 \mathrm{~nm}$ x $50 \mathrm{~nm}$; see [2]) up to the micrometer range with less of the larger particles found in the lymph nodes - an indication for preferred transport of smaller particles.

All in all, we found strong indication for long-term deposition of toxic elements and tattoo pigments in skin and lymph nodes as well as their migration within the human body. Through additional advanced MS-based methodology we were able to reveal simultaneous transport of heavy metals, titanium dioxide and organic pigments from skin to regional lymph nodes. For the pigment copper phthalocyanine green 36 we could demonstrate preferred transport of smaller particles to the lymph nodes. Follow-up experiments will assess the elemental composition of the most popular commercially available black tattoo inks with a focus on the analysis of the organic pigment carbon black.

[1] Laux, P., Tralau, T., Tentschert, J., Blume, A., Al Dahouk, S., Bäumler, W., ... and de Cuyper, C. The Lancet 387.10016 (2016) 395-402.

[2] Schreiver, I., Hesse, B., Seim, C., Castillo-Michel, H., Villanova, J., Laux, P., ... and Luch, A. Scientific reports 7.1 (2017): 11395.

[3] Salomé, M., Cotte, M., Baker, R., Barrett, R., Benseny-Cases, N., Berruyer, G., ... and Gagliardini, E. Journal of Physics: Conference Series. IOP Publishing, (2013) 182004.

[4] Martínez-Criado, G., Villanova, J., Tucoulou, R., Salomon, D., Suuronen, J. P., Labouré, S., ... and Dabin, Y. Journal of synchrotron radiation, 23. Jg., Nr. 1 (2016) 344-352.

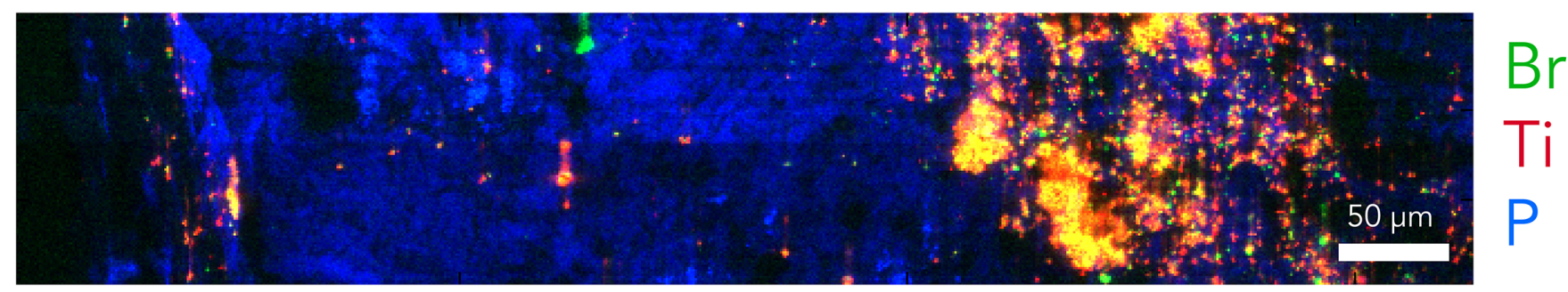

Figure. 1. Lymph node tissue of donor 4 analysed by means of $\mu$-XRF at ID21 at the ESRF with an excitation energy of $5.05 \mathrm{keV}$ and a beam size of $0.3 \mu \mathrm{m} \times 0.7 \mu \mathrm{m}$. All fluorescence intensities are normalized. $\mathbf{P}$ is mostly found in phosphor-rich nuclei and thus gives a vivid image of the lymph node's structure; Br particles from pigment copper phthalocyanine green 36 and Ti particles from white $\mathrm{TiO}_{2}-$ based pigment are mostly co-localized. Particles are found to a lesser degree in the stroma directly beneath the lymph node's capsule (left), however the bulk of the particles is located at a distance of approximately $250 \mu \mathrm{m}$ to the capsule. More $\mu$ - and nano-XRF measurements on skin and lymph node tissue are presented in $[2]$. 